\title{
\begin{tabular}{l|l} 
Mibraries & DSpace@MIT
\end{tabular}
}

\author{
MIT Open Access Articles
}

Clinical utility of microvolt T-wave alternans testing in identifying patients at high or low risk of sudden cardiac death

The MIT Faculty has made this article openly available. Please share how this access benefits you. Your story matters.

Citation: Merchant, Faisal M., Takanori Ikeda, Roberto F.E. Pedretti, Jorge A. Salerno-Uriarte, Theodore Chow, Paul S. Chan, Cheryl Bartone, Stefan H. Hohnloser, Richard J. Cohen, and Antonis A. Armoundas. "Clinical Utility of Microvolt T-Wave Alternans Testing in Identifying Patients at High or Low Risk of Sudden Cardiac Death." Heart Rhythm 9, no. 8 (August 2012): 1256-1264.e2.

As Published: http://dx.doi.org/10.1016/j.hrthm.2012.03.014

Publisher: Elsevier

Persistent URL: http://hdl.handle.net/1721.1/102580

Version: Author's final manuscript: final author's manuscript post peer review, without publisher's formatting or copy editing

Terms of use: Creative Commons Attribution-NonCommercial-NoDerivs License 


\section{Clinical Utility of Microvolt T-wave Alternans Testing In Identifying Patients at High or Low Risk of Sudden Cardiac Death}

Faisal M. Merchant, $\mathrm{MD}^{\star}, \S \S$, Takanori Ikeda, MD, $\mathrm{PhD}^{\dagger}$, Roberto F.E. Pedretti, MD ${ }^{\ddagger}$, Jorge A. Salerno-Uriarte, MD§, Theodore Chow, MDף, Paul S. Chan, MD, MSc ${ }^{\#}$, Cheryl Bartone, MS\|, Stefan H. Hohnloser, MD ${ }^{\dagger \dagger}$, Richard J. Cohen, MD, PhD $\ddagger$, and Antonis A. Armoundas, PhD§§,㧊

*Cardiology Division, Emory University School of Medicine, Atlanta, GA, USA

†Department of Cardiovascular Medicine, Toho University Medical Center, Tokyo, Japan

FDivision of Cardiology, IRCCS Fondazione Salvatore Maugeri, Scientific Institute of Tradate, Italy

$\S$ Department of Heart Sciences, Ospedale di Circolo e Fondazione Macchie, University of Insubria, Varese, Italy

IRegional Medical Center of San Jose, San Jose, CA, USA

\#Mid-America Heart Institute and University of Missouri, Kansas City, MO, USA

"The Heart and Vascular Center at The Christ Hospital, Cincinnati, OH, USA

t†Division of Clinical Electrophysiology, Department of Cardiology, J. W. Goethe University, Frankfurt, Germany

§Cardiology Division, Massachusetts General Hospital, Boston, MA, USA

‡\#Harvard-MIT Division of Health Sciences and Technology, Massachusetts Institute of Technology, Cambridge, MA, USA

\section{Abstract}

Background-Previous studies have demonstrated that microvolt T-wave alternans (MTWA) testing is a robust predictor of ventricular tachyarrhythmias and sudden cardiac death (SCD) in atrisk patients. However, recent studies have suggested that MTWA testing is not as good a predictor of "appropriate" implantable cardioverter-defibrillator (ICD) therapy as it is a predictor of SCD in patients without ICDs.

Objective-We sought to evaluate the utility of MTWA testing for SCD risk stratification in patients without ICDs.

(C) 2012 The Heart Rhythm Society. Published by Elsevier Inc. All rights reserved.

Address for correspondence: Antonis A. Armoundas, PhD, Cardiovascular Research Center, Massachusetts General Hospital, 149

$13^{\text {th }}$ Street, Charlestown, MA 02129, aarmoundas@partners.org.

Conflicts of interest: Theodore Chow - Medtronic Inc., significant

Richard J. Cohen - Cambridge Heart Inc., significant

No other conflicts reported

Publisher's Disclaimer: This is a PDF file of an unedited manuscript that has been accepted for publication. As a service to our customers we are providing this early version of the manuscript. The manuscript will undergo copyediting, typesetting, and review of the resulting proof before it is published in its final citable form. Please note that during the production process errors may be discovered which could affect the content, and all legal disclaimers that apply to the journal pertain. 
Methods-Patient-level data were obtained from five prospective studies of MTWA testing in patients with no history of ventricular arrhythmia or SCD. In these studies, ICDs were implanted in only a minority of patients and patients with ICDs were excluded from the analysis. We conducted a pooled analysis and examined the 2-year risk for SCD based on MTWA test result.

Results-The pooled cohort included 2883 patients. MTWA testing was positive in 856 (30\%), negative in $1627(56 \%)$ and indeterminate in $400(14 \%)$ patients. Among patients with LVEF $\leq$ $35 \%$, annual SCD event rates were $4.0 \%, 0.9 \%$ and $4.6 \%$ among the MTWA positive, negative and indeterminate groups. The SCD rate was significantly lower among patients with a negative MTWA test compared to either the positive or the indeterminate groups ( $<<0.001$ for both comparisons). In patients with $\mathrm{LVEF}>35 \%$, annual SCD event rates were $3.0 \%, 0.3 \%$ and $0.3 \%$ among the MTWA positive, negative and indeterminate groups. The SCD rate associated with a positive test was significantly higher than either the negative $(\mathrm{p}<0.001)$ or the indeterminate groups ( $\mathrm{p}=0.003)$.

Conclusions-In patients without ICDs, MTWA testing is a powerful predictor of SCD. Among patients with LVEF $\leq 35 \%$, a negative MTWA test is associated with a low risk for SCD. Conversely, among patients with LVEF > 35\%, a positive MTWA test identifies patients at significantly heightened SCD risk. These findings may have important implications for refining primary prevention ICD treatment algorithms.

\section{Keywords}

T-wave alternans; arrhythmia; sudden death; risk stratification; electrophysiology; defibrillation

\section{INTRODUCTION}

Implantable cardioverter-defibrillator (ICD) therapy reduces all-cause and arrhythmia specific mortality in patients with a left ventricular ejection fraction (LVEF) $\leq 35 \%$ but without a history of documented sustained ventricular tachyarrhythmias $1,2,3$. However, a number of studies have demonstrated that only a small percentage of patients undergoing primary prevention ICD implantation actually receive appropriate device therapy during long-term follow-up ${ }^{1,2}$. Additionally, ICD implantation is itself associated with morbidity and mortality risk ${ }^{4}$ and thus should be avoided in patients unlikely to benefit from such therapy. It has also been estimated that based on current LVEF criteria for primary prevention ICD therapy, the cost to implant all eligible patients with ICDs would exceed \$30 billion, a figure which would roughly equal the annual total expenditure for all heart failure related care in this country ${ }^{5}$. Based on these observations, it is clear that current ICD eligibility criteria are unsustainable from a healthcare system perspective and more robust tools are necessary to identify which patients are most, or least, likely to benefit from ICD therapy.

Microvolt T-wave alternans (MTWA) testing using the spectral method has emerged as a predictor of ventricular tachyarrhythmic events (VTE) and sudden cardiac death (SCD) in at-risk populations. Prospective studies, primarily in patients without ICDs, have demonstrated that a positive MTWA test result indicates a high level of risk while a negative MTWA test confers a very low level of risk ${ }^{6-9}$. However, in some recent studies involving patient populations with a higher percentage of implanted ICDs, MTWA test result was not found to be as good a predictor of "appropriate" ICD therapy as it is a predictor of VTE/ SCD in patients without ICDs ${ }^{10}$. This observation has been attributed to the fact that many "appropriate" ICD therapies treat arrhythmias that would have self-terminated or that ICDs may induce arrhythmias that they subsequently treat ${ }^{10-12}$. In light of these findings, it is plausible that MTWA testing is best utilized for patients who do not already have ICDs in order to determine whether they are at risk and should be considered for ICD therapy. In a 
prospective study of patients with LVEF $\leq 35 \%$, ICD therapy reduced annual mortality by approximately $50 \%$ among patients with a non-negative MTWA test and provided no benefit among those with a negative test, suggesting the efficacy of MTWA testing in predicting the effectiveness of ICD therapy ${ }^{13}$.

The ability to accurately predict risk of VTE/SCD is a crucial step in identifying which patients are most, or least, likely to benefit from ICD therapy. In order to address this question, we assessed the predictive utility of MTWA testing on arrhythmia specific endpoints by conducting a pooled patient-level analysis of prospective studies of MTWA in patients without ICDs.

\section{METHODS}

\section{Study Design}

We identified prospective clinical trials (for more information, please see the On-line Supplement) involving at least 100 patients in which MTWA testing using the spectral analytic method was used to predict the risk of SCD and all-cause mortality with at least 12 month follow-up. In order to minimize the impact of ICD therapy on study endpoints, we excluded studies where $\geq 15 \%$ of the patients had ICDs implanted at baseline or $\geq 15 \%$ of the total arrhythmic outcome events were due to "appropriate" ICD therapy ${ }^{10}$. Additionally, in order to further minimize the impact of ICD therapy, patients with ICDs from the included studies were excluded from the final pooled cohort analysis.

We obtained patient level data from the authors of the five cohorts included in this pooled analysis. The baseline characteristics and details of each of the five studies have been published previously $8,9,14-16$ and are summarized in Table 1 . All studies utilized MTWA testing with the spectral method ${ }^{17}$ (CH 2000 system, Cambridge Heart, Bedford, MA, USA) and the results of each MTWA test (positive, negative or indeterminate) were classified by the investigators within each study based on established criteria ${ }^{18}$. Indeterminate tests were further sub-classified based on cause into one of four groups: 1) excessive ventricular ectopy during exercise, 2$)$ unsustained alternans ( $<1$ minute), 3 ) inability to reach target HR ( $<105$ beats/min), or 4) excessive noise.

\section{Study Endpoints}

All arrhythmic events and mortality endpoints were adjudicated by the study investigators based on the specific definitions used within each study protocol ${ }^{8,9,14-16}$. For the purpose of this pooled analysis, the primary endpoint was arrhythmic mortality/SCD at 24 months. The secondary endpoint was all-cause mortality at 24 months. In order to compare endpoints across different studies with varying follow-up periods, outcome data were converted to annual event rates based on data collected over a 24 -month period. The annual event rate was calculated using an exponential survival model which assumes that individuals have a constant probability of experiencing the outcome event per unit time ${ }^{10}$. All endpoints are reported as annual event rates derived over a 24-month follow-up period.

\section{Statistical Analysis}

We performed a pooled analysis of patient-level data from the five studies meeting our inclusion criteria to assess the risk of SCD based on MTWA test result. We initially performed preliminary analysis using both pooled and meta-analytic techniques and found that the estimated event rates and p-values across sub-groups were similar using both techniques (data not shown). However, we ultimately made the decision to proceed with a pooled analysis based on two limitations of the meta-analytic technique: 1) inability to estimate event rates across studies using meta-analysis in sub-groups with relatively few 
total events and 2) lack of a validated approach for generating summary survival curves with meta- techniques.

The time course of the primary and secondary endpoints, stratified by MTWA result, was estimated by Kaplan-Meier time to first event curves. The association between MTWA test result and the primary and secondary endpoints was assessed by Kaplan-Meier product-limit estimates and tested with the log-rank test.

Continuous variables are presented as mean \pm standard deviation and categorical data are summarized as frequencies and percentages. Comparisons across groups were performed using the one-way ANOVA or Chi-square test of independence, as appropriate. For all comparisons, a $\mathrm{p}<0.05$ was considered to be statistically significant. Analysis was performed using STATISTICA software (Statsoft, Inc., Tulsa, OK).

\section{RESULTS}

\section{Primary Endpoint - Arrhythmic Mortality/Sudden Cardiac Death based on MTWA result}

The baseline characteristics of patients included in the final pooled cohort, stratified by the study of origin, are listed in Table 1. A total of 479 patients implanted with ICDs were excluded from the final analysis yielding a pooled study cohort of 2883 . Overall, mean age was $62.8 \pm 11.0$ years, mean LVEF was $44.2 \% \pm 15.6 \%$ and approximately $80 \%$ of patients had underlying ischemic heart disease.

Results of MTWA testing were positive in 856 (30\%), negative in $1627(56 \%)$ and indeterminate in 400 patients (14\%). Patients with indeterminate MTWA test results were older than patients with either positive or negative results, whereas patients with negative MTWA results had higher baseline LVEF and were more likely to have ischemic heart disease than either the positive or the indeterminate groups (Table 2). Among patients with indeterminate MTWA test results, the most common cause of indeterminacy was excessive ventricular ectopy during exercise $(59 \%)$, followed by inadequate HR $(21 \%)$, noise (11\%) and unsustained alternans (6\%) (Figure 1). Only 3\% of indeterminate studies could not be further sub-classified based on specific etiology.

The primary endpoint of arrhythmic mortality/sudden cardiac death occurred in 80 patients at the 24-month time point. The annual event rate for SCD among patients with positive, negative and indeterminate MTWA test results was 3.5\%, $0.4 \%$ and $2.5 \%$, respectively (Table 3). The Kaplan-Meier event-free survival curves for the primary endpoint stratified by MTWA test result are shown in Figure 2. The SCD event rate was significantly higher for patients with both positive and indeterminate MTWA test results when compared to negative patients ( $\mathrm{p}<0.001$ for both comparisons) whereas the event rates were not significantly different between the positive and the indeterminate groups $(p=0.19)$. When stratified by cause of indeterminacy, the annual SCD event rates among patients who were indeterminate due to excessive ectopy $(1.9 \%)$ or inadequate HR $(4.1 \%)$ were also significantly higher than the negative cohort and not significantly differently than the event rate among patients with positive results. In contrast, although the SCD event rates among patients who were indeterminate due to noise (1.4\%) and unsustained alternans (no events) appeared low, because of the small numbers of patients in these groups, the event rates were not statistically different than either the MTWA positive or negative groups (Table 3).

\section{Primary Endpoint - Arrhythmic Mortality/Sudden Cardiac Death based on LVEF and MTWA result}

The pooled study cohort was further sub-classified based on LVEF. Among patients with LVEF $\leq 35 \%$, the annual SCD event rates for the positive, negative and indeterminate 
groups were $4.0 \%, 0.9 \%$ and $4.6 \%$, respectively. Kaplan-Meier analysis demonstrated that among patients with LVEF $\leq 35 \%$, the pattern of SCD event-free survival was similar to the overall cohort with event-free survival being significantly lower among patients with positive and indeterminate test results compared to the MTWA negative group (Figure 3). There was no significant difference in SCD event-free survival between positives and indeterminates among patients with LVEF $\leq 35 \%$. Additionally, when stratified by the cause of indeterminacy, SCD events rates for patients with excessive ectopy (4.2\%) and inadequate HR $(7.4 \%)$ were significantly worse than the negative group but not significantly different than the MTWA-positive patients (Table 3).

Among patients with LVEF $>35 \%$, SCD annual event rates stratified by MTWA test result were: positive $(3.0 \%)$, negative $(0.3 \%)$ and indeterminate $(0.3 \%)$. Among patients in this category, survival free of SCD was significantly worse for MTWA-positive patients than either the MTWA-negative $(\mathrm{p}<0.001)$ or the MTWA-indeterminate $(\mathrm{p}=0.003)$ groups, whereas event-free survival was not significantly different between the negative and indeterminate groups with LVEF > 35\% ( $\mathrm{p}=0.801)$ (Figure 4). There was only one SCD event among patients with indeterminate MTWA test result and LVEF $>35 \%$ and therefore, events rates were uniformly low regardless of the cause of indeterminacy although statistical power among the indeterminate sub-groups was limited (Table 3).

\section{Secondary Endpoint - All-Cause Mortality}

With regard to the secondary endpoint of all-cause mortality, the annual event rates for the entire pooled cohort among patients with positive, negative and indeterminate test results were $7.8 \%, 1.5 \%$ and $6.8 \%$, respectively (Table 4 ). The event rates were significantly higher for patients with positive and indeterminate studies when compared to those with negative studies ( $p<0.001$ for both comparisons) whereas there was no significant difference in allcause mortality rate between positives and indeterminates $(\mathrm{p}=0.40)$. Among causes of indeterminacy, the event rates among patients with excessive ectopy (6.4\%), inadequate HR $(8.7 \%)$ and noise $(5.3 \%)$ were all significantly higher than the rate among negative patients and not significantly different than the positive patients.

When stratified by LVEF, the trends in all-cause mortality were similar to the trends in SCD event rates. Specifically, among patients with LVEF $\leq 35 \%$, the all-cause mortality event rates in the positive $(9.7 \%)$ and indeterminate $(12.2 \%)$ groups were significantly higher than the negative cohort $(4.2 \%)$ ( $\mathrm{p}<0.001$ for both comparisons). In contrast, in the LVEF > 35\% cohort, the all-cause mortality rate among MTWA positive patients $(6.2 \%)$ was significantly higher than the rate among the negative $(0.7 \%)$ or indeterminate $(1.1 \%)$ groups whereas there was no significant difference in all-cause mortality rates between the MTWAindeterminate and MTWA-negative patients $(\mathrm{p}=0.25)$.

\section{DISCUSSION}

Several important conclusions can be drawn from this pooled analysis of patients with heterogeneous levels of risk for SCD but without ICDs. First, a negative MTWA test result clearly identifies a population of patients at very low risk of SCD during the ensuing 24 months, regardless of LV function (annual event rate $0.9 \%$ in patients with LVEF $\leq 35 \%$ and $0.3 \%$ with LVEF > 35\%). This finding suggests that patients with a negative MTWA test, even with LVEF $\leq 35 \%$, are likely to be at sufficiently low arrhythmic risk that they may not benefit from prophylactic ICD therapy. Second, even among patients with LVEF > $35 \%$, a positive MTWA test identifies a cohort at significantly heightened risk of SCD for whom targeted therapies may be beneficial to mitigate arrhythmic risk. Lastly, the risk of SCD among patients with indeterminate MTWA results is highly dependent on ejection fraction. Among patients with $\mathrm{LVEF} \leq 35 \%$, an indeterminate MTWA test - particularly 
among those who are indeterminate due to excessive ectopy or inadequate $\mathrm{HR}$ - predicts an increased risk of SCD at least as well as a positive test. In contrast, an indeterminate MTWA test in patients with LVEF > 35\% does not predict an increased risk of SCD and therefore, these patients should not be grouped with patients who test positive.

Several important aspects of our work should be highlighted. First, in contrast to many earlier MTWA studies, we used SCD/arrhythmic mortality, rather than all-cause mortality, as the primary endpoint. Although we report data on all-cause mortality, SCD is likely to be a far more germane endpoint in terms of making decisions about ICD therapy. Second, we specifically excluded patients with ICDs from our analysis. There has been significant concern about the use of "appropriate" ICD therapy as an endpoint in recent clinical trials ${ }^{11}$ and it is widely recognized that "appropriate" ICD therapy overestimates the true incidence of SCD. In fact, a high percentage of implanted ICDs has been suggested as an explanation for why several recent studies have failed to demonstrate the predictive value of MTWA testing ${ }^{10}$. We designed our study specifically to avoid this potential source of confounding. Lastly, and perhaps most importantly, this cohort is particularly unique in that we were able to assemble a large group of patients who were at-risk of SCD (based on current evidence) but because they were enrolled in studies prior to the era of contemporary ICD guidelines ${ }^{19}$, they were not routinely implanted with ICDs. Therefore, we were able to analyze the natural history of SCD risk in a large cohort of patients. It is highly unlikely that such a cohort will ever be assembled again, outside of a randomized clinical trial, since most such patients would now be deemed ICD candidates.

Our pooled cohort represents a heterogeneous group of patients including both ischemic and non-ischemic cardiomyopathy with a wide range of LVEF. From a clinical perspective, we believe the predictive capability of MTWA testing across a heterogeneous cohort represents an important strength of this study. The recent report from the NHLBI/HRS working group on Sudden Cardiac Death Prediction and Prevention ${ }^{20}$ emphasizes the need to find other markers of SCD risk, beyond LVEF, which can be applied to the large group of patients currently eligible for ICD therapy to refine risk prediction and improve ICD allocation. In that light, the purpose of this paper is to examine the utility of MTWA testing as a screening test to risk stratify patients and refine decision making about ICD therapy.

\section{MTWA Testing to Guide ICD Therapy in Patients with Ejection Fraction $\leq \mathbf{3 5 \%}$}

Drawing largely from the Multicenter Automatic Defibrillator Implantation Trial II (MADIT II) ${ }^{1}$ and the Sudden Cardiac Death in Heart Failure Trial (SCD-HeFT) ${ }^{2}$, current guidelines identify candidates for primary prevention ICD therapy based primarily on LV ejection fraction. However, the broad application of MADIT II and SCD-HeFT criteria has confirmed that only a small percentage of patients actually receive appropriate ICD therapy, highlighting the need for more robust tools to identify which patients are, and which patients are not, likely to benefit from ICDs ${ }^{21}$.

Our findings from a large cohort of patients demonstrate that even among patients with LVEF $\leq 35 \%$, those who are MTWA negative are at such low risk of arrhythmic mortality that they are unlikely to benefit from prophylactic ICD therapy. Trials demonstrating the mortality benefit of prophylactic ICD therapy in patients with reduced LVEF were conducted in patient populations with far higher event rates than those observed in such patients who test MTWA negative (e.g. the annual mortality rates in the control arms of MADIT II ${ }^{1}$ and SCD-HeFT ${ }^{2}$ were $13.2 \%$ and $9.0 \%$ respectively, versus the $4.2 \%$ annual mortality reported here in MTWA negative patients with LVEF $\leq 35 \%$ ). Furthermore, a prospective study ${ }^{13}$ demonstrated that ICD therapy reduced annual mortality only in patients with a non-negative MTWA test and not in patients with a negative MTWA test. 
Thus, there is an absence of evidence demonstrating that prophylactic ICD therapy is beneficial to MTWA negative patients.

In the present study, $38.5 \%$ of LVEF $\leq 35 \%$ patients had a negative MTWA test. The ability to safely withhold device therapy from these patients represents a major opportunity to reduce unnecessary exposure to an invasive treatment with well-established short- and longterm complications ${ }^{4}$ while at the same time improving resource allocation and reducing the cost burden to the healthcare system. These data strongly suggest the need for future, prospective studies designed specifically to assess the value of ICD therapy in patients with a negative MTWA test result.

\section{MTWA Testing for SCD Risk Stratification in Patients with Ejection Fraction > 35\%}

Although patients with LVEF $\leq 35 \%$ are at significantly increased risk of SCD, the great majority of SCDs occur in patients with LVEF $>35 \% 22$. Our findings suggest that among patients with LVEF > 35\%, a positive MTWA test identifies a group with a 10-fold higher risk of SCD (annual event rate 3.0\% vs. $0.3 \%$ among patients who are MTWA negative or indeterminate). In patients with preserved LV systolic function but with other risk factors for SCD (i.e. coronary artery disease or other forms of structural heart disease), MTWA testing may provide a means for non-invasively identifying patients at heightened arrhythmic risk. Prospective studies will be necessary to determine if life-style interventions, pharmacologic and/or device therapies ${ }^{23}$ are capable of mitigating SCD risk among patients with LVEF > $35 \%$ and a positive MTWA test.

\section{Prognosis of Patients with Indeterminate MTWA Test Results}

One challenge in interpreting MTWA trials is appropriate classification of patients with indeterminate test results. Early studies suggested that the prognosis of patients who are MWTA indeterminate does not differ significantly from those who are positive and therefore, these two groups have traditionally been classified in a single "non-negative" category ${ }^{18}$. Two studies have looked specifically at the impact of reclassifying studies based on the cause of indeterminacy 24,25 . Our findings are consistent with these prior studies in demonstrating that patients who are indeterminate due to excessive ectopy or inadequate HR are at significantly higher risk than patients who test negative. However, our findings add the important caveat that the prognosis associated with an indeterminate MTWA test result is highly dependent on LV function. Specifically, patients who are indeterminate with LVEF $\leq 35 \%$ (particularly those who are indeterminate due to excessive ectopy or inadequate HR) have a heightened risk of SCD and can be appropriately grouped with patients who are MTWA-positive. In contrast, the risk of SCD among patients with LVEF > 35\% who are indeterminate is substantially lower than those who test positive and not different than those who test negative. In patients with LVEF > 35\%, only a positive MTWA test confers an increased risk. Two important differences should be noted between our study and prior studies investigating the prognosis of indeterminate MTWA tests. First, in contrast to prior studies, our study used SCD as the primary endpoint, rather than allcause mortality. Second, previous studies have included patients with ICDs 24,25 whereas our study specifically excluded patients with ICDs from the final analysis.

\section{Study Limitations}

Several limitations of our study should be noted. First, the classification of MTWA test results was performed by the investigators of each individual study rather than being rereviewed centrally. However, specific criteria have been established for determining positive and negative MTWA test results ${ }^{18}$ and these criteria were used by the investigators in each of the five source studies. Second, the definitions used for adjudicating clinical endpoints (i.e. arrhythmic vs. non-arrhythmic mortality) were slightly different in each of the cohorts 
included in this pooled analysis and may have introduced some inconsistency when comparing across studies. Third, although we were able to incorporate the impact of LVEF on SCD risk, we did not have data on clinical heart failure symptoms (systolic or diastolic) and therefore, were unable to assess the impact of heart failure symptom severity. Lastly, due to relatively small numbers of events in certain sub-groups, we chose a pooled cohort methodology rather than formal meta-analysis; however, the pooled cohort does not account for between-study variability, which is a limitation of this technique.

\section{Conclusions}

Our findings demonstrate that in a large pooled cohort of patients at varying levels of arrhythmic risk but without ICDs, MTWA testing is a powerful predictor of SCD. In patients regardless of LVEF, a negative MTWA test result identifies a cohort of patients with very low risk of SCD and a positive MTWA test identifies a patient group at high risk of SCD. In contrast, patients with an indeterminate MTWA test result have a higher risk of SCD only if the LVEF is $\leq 35 \%$. These findings may have important implications for refining primary prevention ICD treatment algorithms and improving resource allocation.

\section{Acknowledgments}

Funding sources: none

\section{Abbreviations}

$\begin{array}{ll}\text { ICD } & \text { Implantable cardioverter-defibrillator } \\ \text { LVEF } & \text { left ventricular ejection fraction } \\ \text { MTWA } & \text { Microvolt T-wave alternans } \\ \text { VTE } & \text { ventricular tachyarrhythmic events } \\ \text { SCD } & \text { sudden cardiac death } \\ \text { HR } & \text { heart rate }\end{array}$

\section{References}

1. Moss AJ, Zareba W, Hall WJ, et al. Prophylactic implantation of a defibrillator in patients with myocardial infarction and reduced ejection fraction. N Engl J Med. 2002; 346:877-883. [PubMed: 11907286]

2. Bardy GH, Lee KL, Mark DB, et al. Amiodarone or an implantable cardioverter-defibrillator for congestive heart failure. N Engl J Med. 2005; 352:225-237. [PubMed: 15659722]

3. Merchant FM, Armoundas AA. Role of substrate and triggers in the genesis of cardiac alternans, from the myocyte to the whole heart: implications for therapy. Circulation. 2012; 125:539-549. [PubMed: 22271847]

4. Reynolds MR, Cohen DJ, Kugelmass AD, et al. The frequency and incremental cost of major complications among medicare beneficiaries receiving implantable cardioverter-defibrillators. J Am Coll Cardiol. 2006; 47:2493-2497. [PubMed: 16781379]

5. Stevenson LW. Projecting heart failure into bankruptcy in 2012? Am Heart J. 161:1007-1011. [PubMed: 21641344]

6. Bloomfield DM, Bigger JT, Steinman RC, et al. Microvolt T-wave alternans and the risk of death or sustained ventricular arrhythmias in patients with left ventricular dysfunction. J Am Coll Cardiol. 2006; 47:456-463. [PubMed: 16412877]

7. Hohnloser SH, Ikeda T, Bloomfield DM, Dabbous OH, Cohen RJ. T-wave alternans negative coronary patients with low ejection and benefit from defibrillator implantation. Lancet. 2003; 362:125-126. [PubMed: 12867114] 
8. Ikeda T, Yoshino H, Sugi K, et al. Predictive value of microvolt T-wave alternans for sudden cardiac death in patients with preserved cardiac function after acute myocardial infarction: results of a collaborative cohort study. J Am Coll Cardiol. 2006; 48:2268-2274. [PubMed: 17161258]

9. Salerno-Uriarte JA, De Ferrari GM, Klersy C, et al. Prognostic value of T-wave alternans in patients with heart failure due to nonischemic cardiomyopathy: results of the ALPHA Study. J Am Coll Cardiol. 2007; 50:1896-1904. [PubMed: 17980258]

10. Hohnloser SH, Ikeda T, Cohen RJ. Evidence regarding clinical use of microvolt T-wave alternans. Heart Rhythm. 2009; 6:S36-44. [PubMed: 19168396]

11. Ellenbogen KA, Levine JH, Berger RD, et al. Are implantable cardioverter defibrillator shocks a surrogate for sudden cardiac death in patients with nonischemic cardiomyopathy? Circulation. 2006; 113:776-782. [PubMed: 16461817]

12. Germano JJ, Reynolds M, Essebag V, Josephson ME. Frequency and causes of implantable cardioverter-defibrillator therapies: is device therapy proarrhythmic? Am J Cardiol. 2006; 97:1255-1261. [PubMed: 16616037]

13. Chow T, Kereiakes DJ, Bartone C, et al. Microvolt T-wave alternans identifies patients with ischemic cardiomyopathy who benefit from implantable cardioverter-defibrillator therapy. J Am Coll Cardiol. 2007; 49:50-58. [PubMed: 17207722]

14. Klingenheben T, Zabel M, D'Agostino RB, Cohen RJ, Hohnloser SH. Predictive value of T-wave alternans for arrhythmic events in patients with congestive heart failure [letter]. Lancet. 2000; 356:651-652. [PubMed: 10968440]

15. Ikeda T, Saito H, Tanno K, et al. T-wave alternans as a predictor for sudden cardiac death after myocardial infarction. Am J Cardiol. 2002; 89:79-82. [PubMed: 11779531]

16. Chan PS, Nallamothu BK, Spertus JA, et al. Impact of age and medical comorbidity on the effectiveness of implantable cardioverter-defibrillators for primary prevention. Circ Cardiovasc Qual Outcomes. 2009; 2:16-24. [PubMed: 20031808]

17. Rosenbaum DS, Jackson LE, Smith JM, et al. Electrical alternans and vulnerability to ventricular arrhythmias. N Engl J Med. 1994; 330:235-241. [PubMed: 8272084]

18. Bloomfield DM, Hohnloser SH, Cohen RJ. Interpretation and classification of microvolt $\mathrm{T}$ wave alternans tests. J Cardiovasc Electrophysiol. 2002; 13:502-512. [PubMed: 12030535]

19. Epstein AE, DiMarco JP, Ellenbogen KA, et al. ACC/AHA/HRS 2008 Guidelines for DeviceBased Therapy of Cardiac Rhythm Abnormalities: a report of the American College of Cardiology/American Heart Association Task Force on Practice Guidelines (Writing Committee to Revise the ACC/AHA/NASPE 2002 Guideline Update for Implantation of Cardiac Pacemakers and Antiarrhythmia Devices): developed in collaboration with the American Association for Thoracic Surgery and Society of Thoracic Surgeons. Circulation. 2008; 117:e350-408. [PubMed: 18483207]

20. Fishman GI, Chugh SS, Dimarco JP, et al. Sudden cardiac death prediction and prevention: report from a National Heart, Lung, and Blood Institute and Heart Rhythm Society Workshop. Circulation. 2010; 122:2335-2348. [PubMed: 21147730]

21. Armoundas AA, Hohnloser SH, Ikeda T, Cohen RJ. Can microvolt T-wave alternans testing reduce unnecessary defibrillator implantation? Nat Clin Pract Cardiovasc Med. 2005; 2:522-528. [PubMed: 16186850]

22. Stecker EC, Vickers C, Waltz J, et al. Population-based analysis of sudden cardiac death with and without left ventricular systolic dysfunction: two-year findings from the Oregon Sudden Unexpected Death Study. J Am Coll Cardiol. 2006; 47:1161-1166. [PubMed: 16545646]

23. Zipes DP, Camm AJ, Borggrefe M, et al. ACC/AHA/ESC 2006 guidelines for management of patients with ventricular arrhythmias and the prevention of sudden cardiac death--executive summary: A report of the American College of Cardiology/American Heart Association Task Force and the European Society of Cardiology Committee for Practice Guidelines (Writing Committee to Develop Guidelines for Management of Patients with Ventricular Arrhythmias and the Prevention of Sudden Cardiac Death) Developed in collaboration with the European Heart Rhythm Association and the Heart Rhythm Society. Eur Heart J. 2006; 27:2099-2140. [PubMed: 16923744] 
24. Kaufman ES, Bloomfield DM, Steinman RC, et al. "Indeterminate" microvolt T-wave alternans tests predict high risk of death or sustained ventricular arrhythmias in patients with left ventricular dysfunction. J Am Coll Cardiol. 2006; 48:1399-1404. [PubMed: 17010802]

25. Chan PS, Bartone C, Booth T, Kereiakes D, Chow T. Prognostic implication of redefining indeterminate microvolt T-wave alternans studies as abnormal or normal. Am Heart J. 2007; 153:523-529. [PubMed: 17383288] 


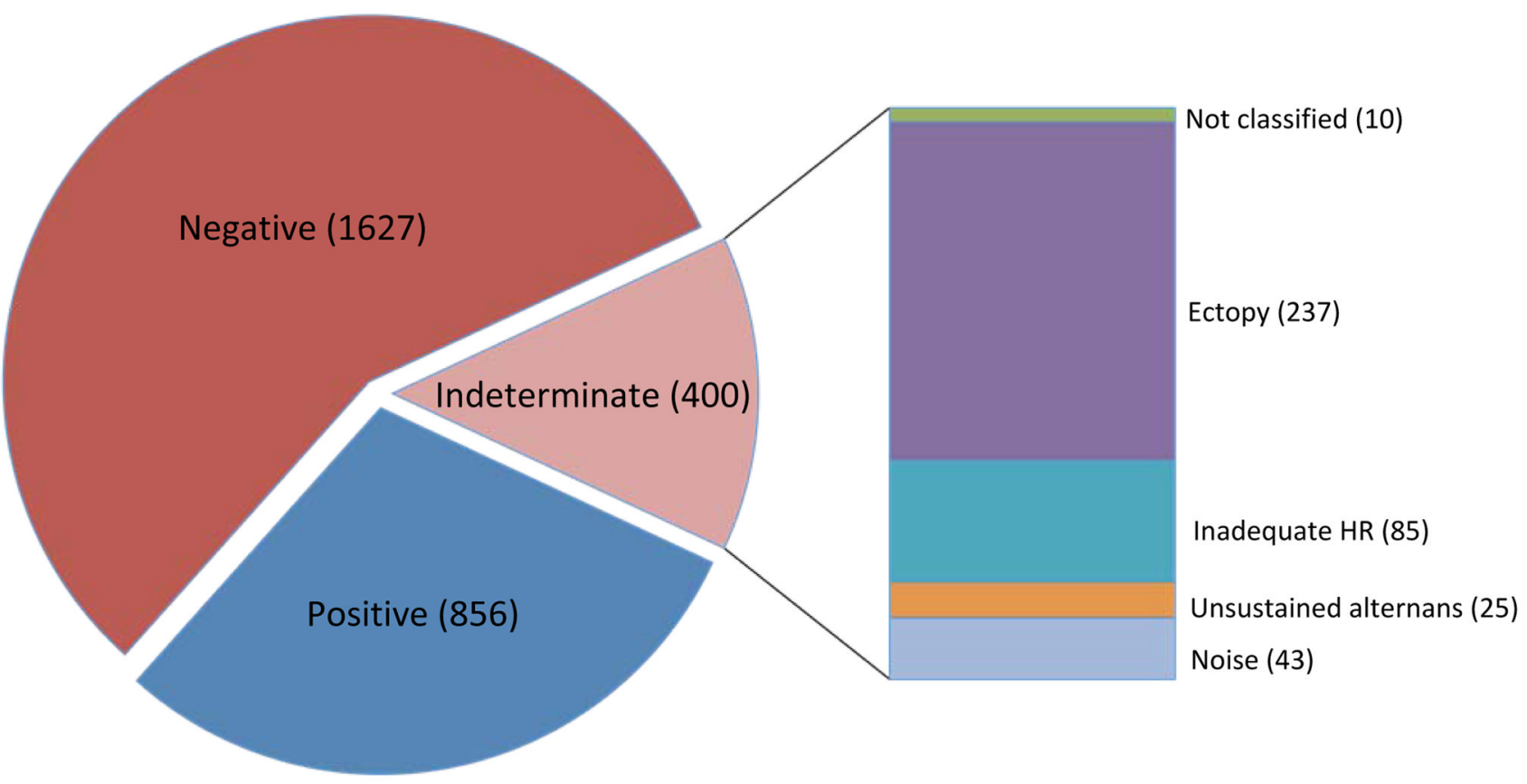

Figure 1. MTWA test results across the entire pooled cohort including sub-classification based on cause of indeterminacy 


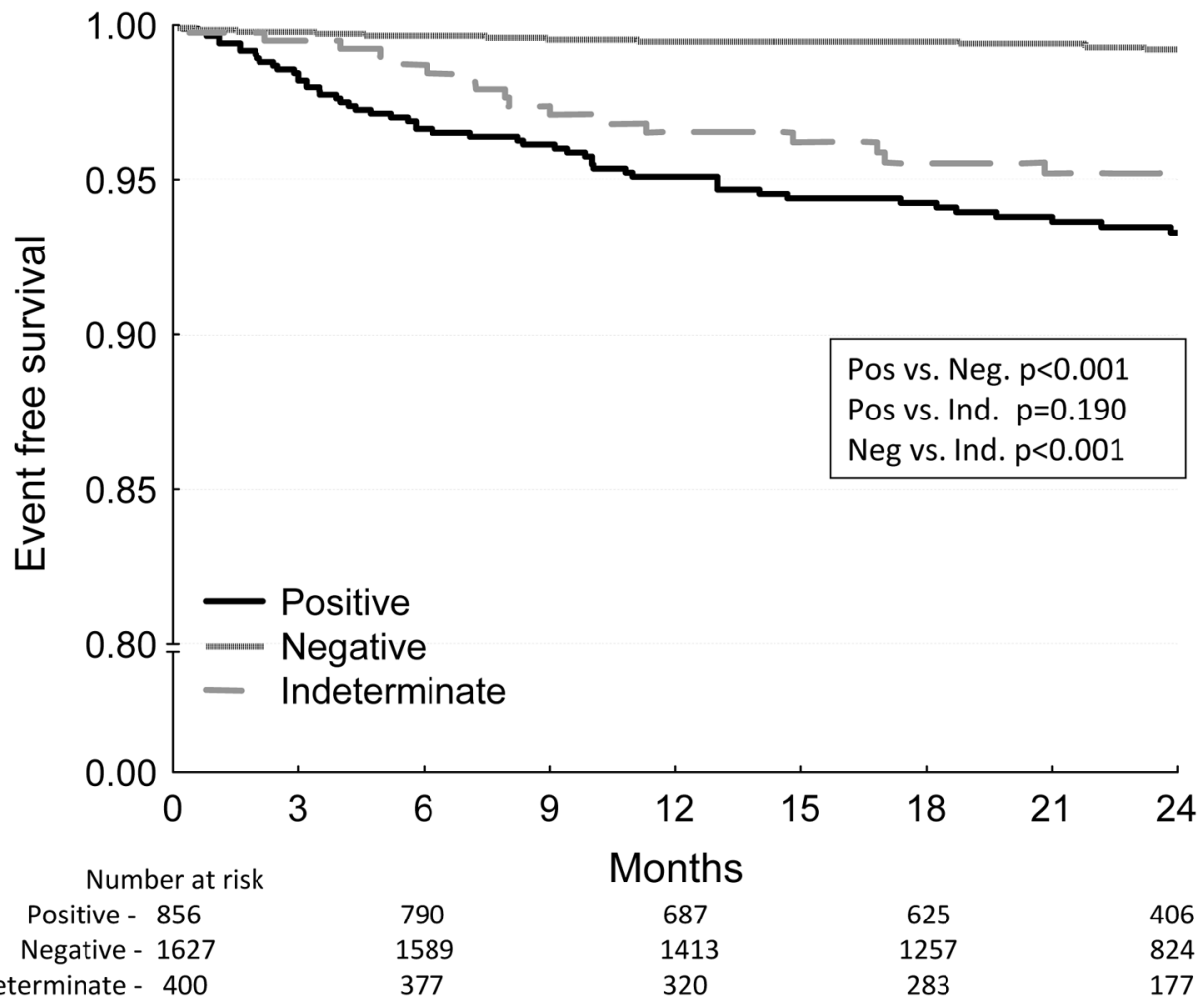

Figure 2. Kaplan-Meier event-free survival curves for the primary endpoint of arrhythmic mortality/sudden cardiac death stratified by MTWA test result for the entire pooled cohort Patients with positive or indeterminate MTWA test result demonstrate significantly lower event-free survival compared to patients with negative MTWA test results. There is no significant difference in event-free survival between patients with positive and indeterminate test results. All p values are generated by the log-rank test. 


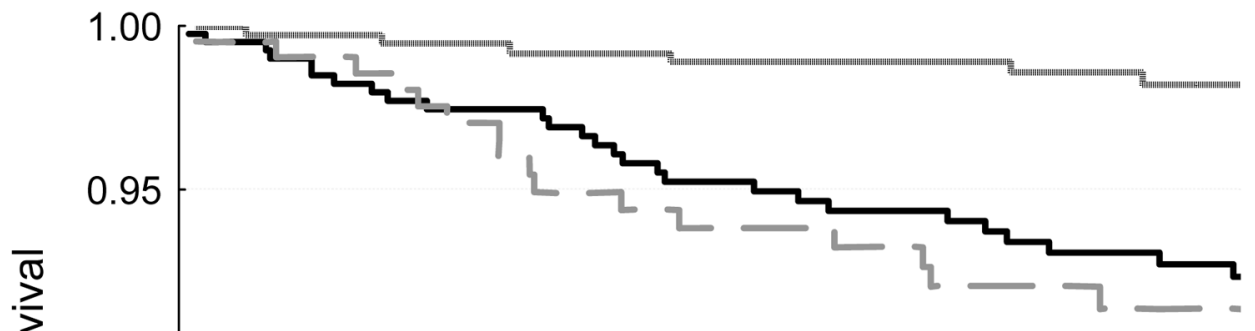

Pos vs. Neg. $p<0.001$

Pos vs. Ind. $p=0.632$

Neg vs. Ind. $p<0.001$

華 0.85

Ш

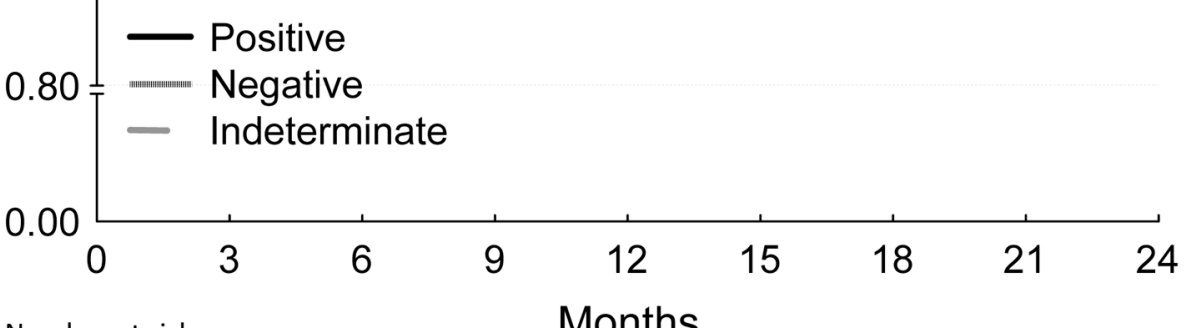

Number at risk Positive - 406

Figure 3. Kaplan-Meier event-free survival curves for the primary endpoint of arrhythmic mortality/sudden cardiac death stratified by MTWA test result among patients with LVEF $\leq$ $35 \%$

Similar to the pattern observed in the whole cohort, event-free survival is significantly lower among patients with positive or indeterminate MTWA test results, whereas there is no significant difference in survival between the positive and indeterminate groups. All $\mathrm{p}$ values are generated by the log-rank test. 


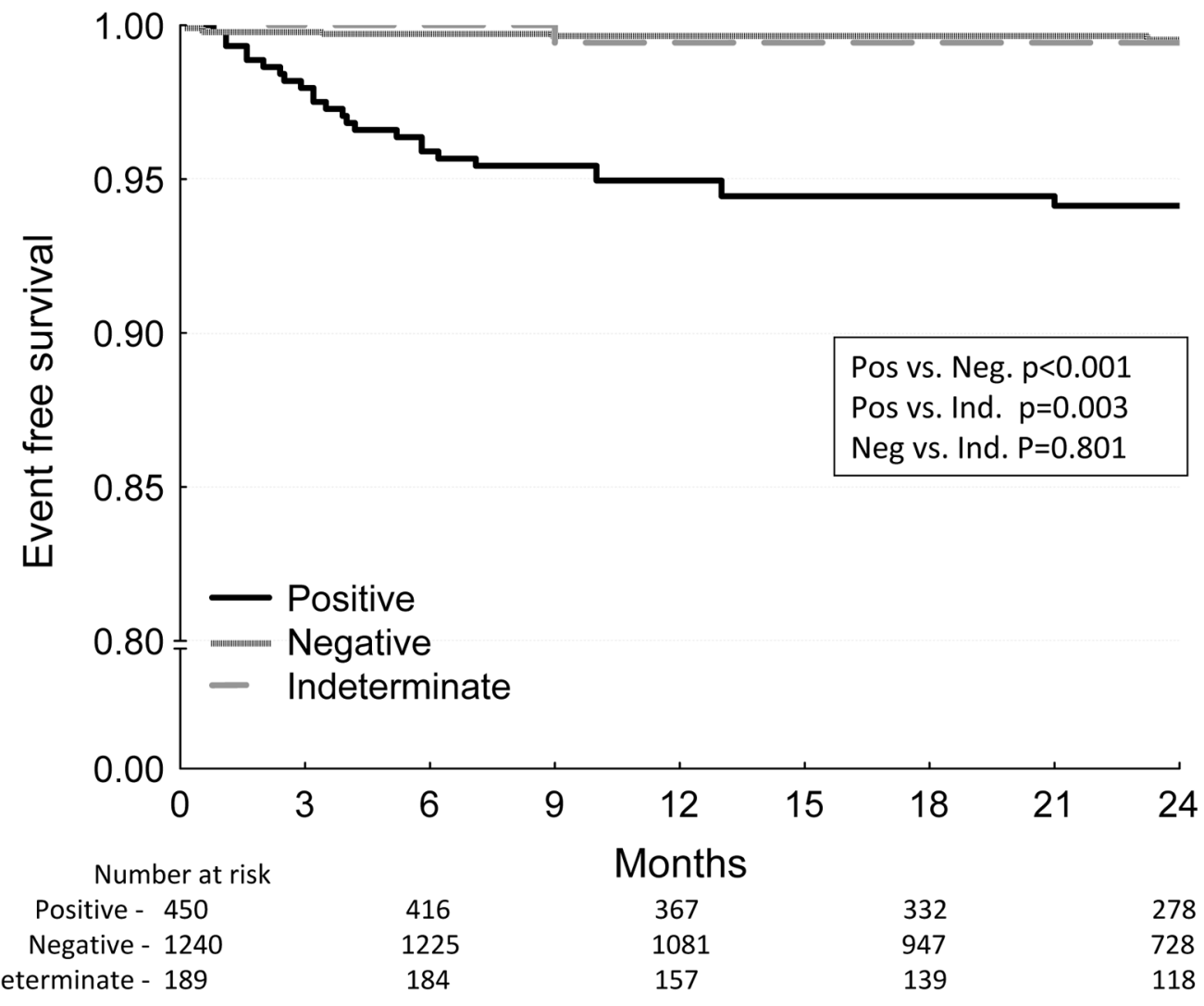

Figure 4. Kaplan-Meier event-free survival curves for the primary endpoint of arrhythmic mortality/sudden cardiac death stratified by MTWA test result among patients with LVEF > $35 \%$

Event-free survival is significantly lower in the MTWA positive group than either the negative or indeterminate groups. In contrast, there is no significant difference in event-free survival between the negative and indeterminate groups. All p values are generated by the log-rank test. 


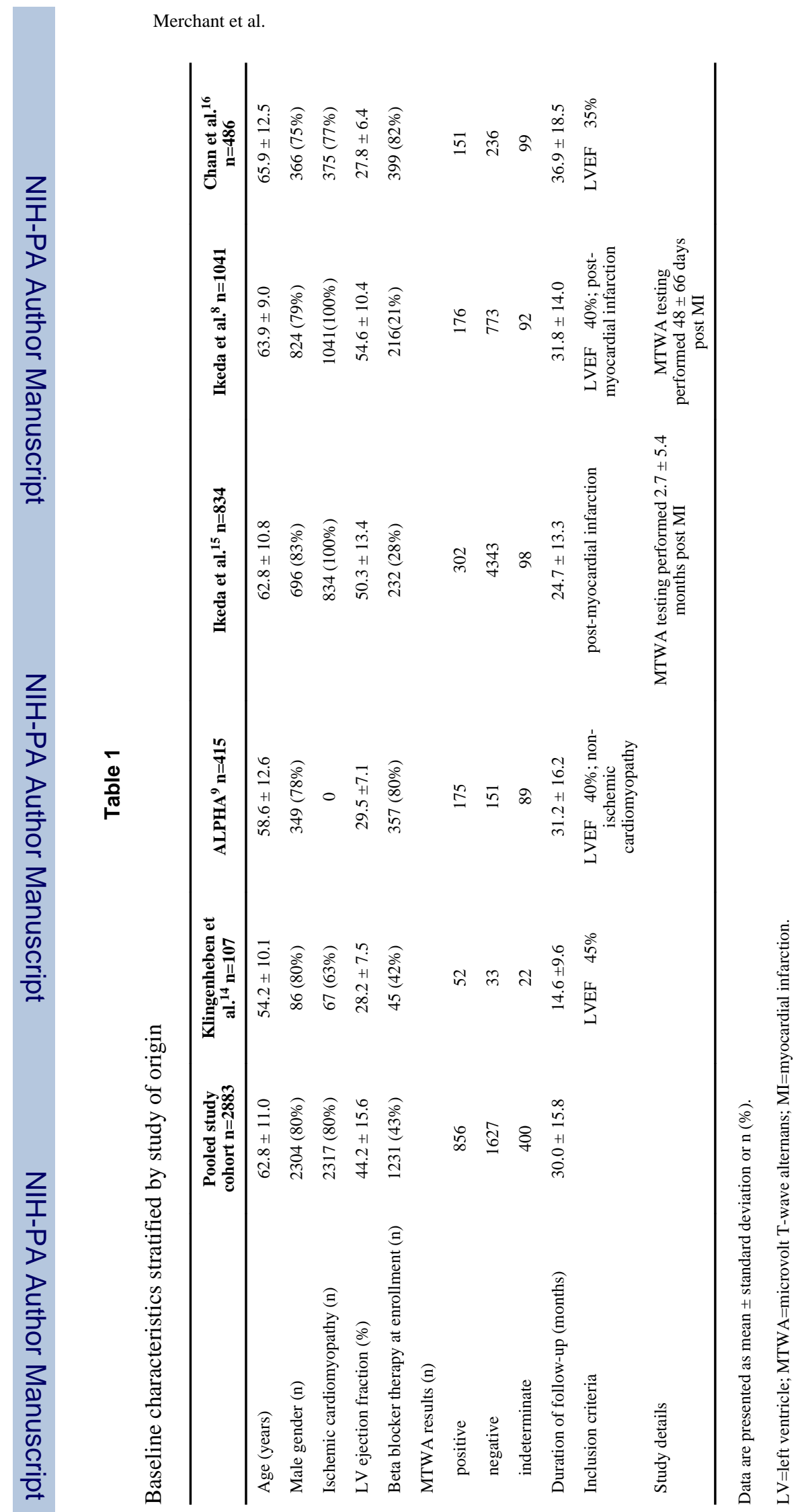

Page 15 


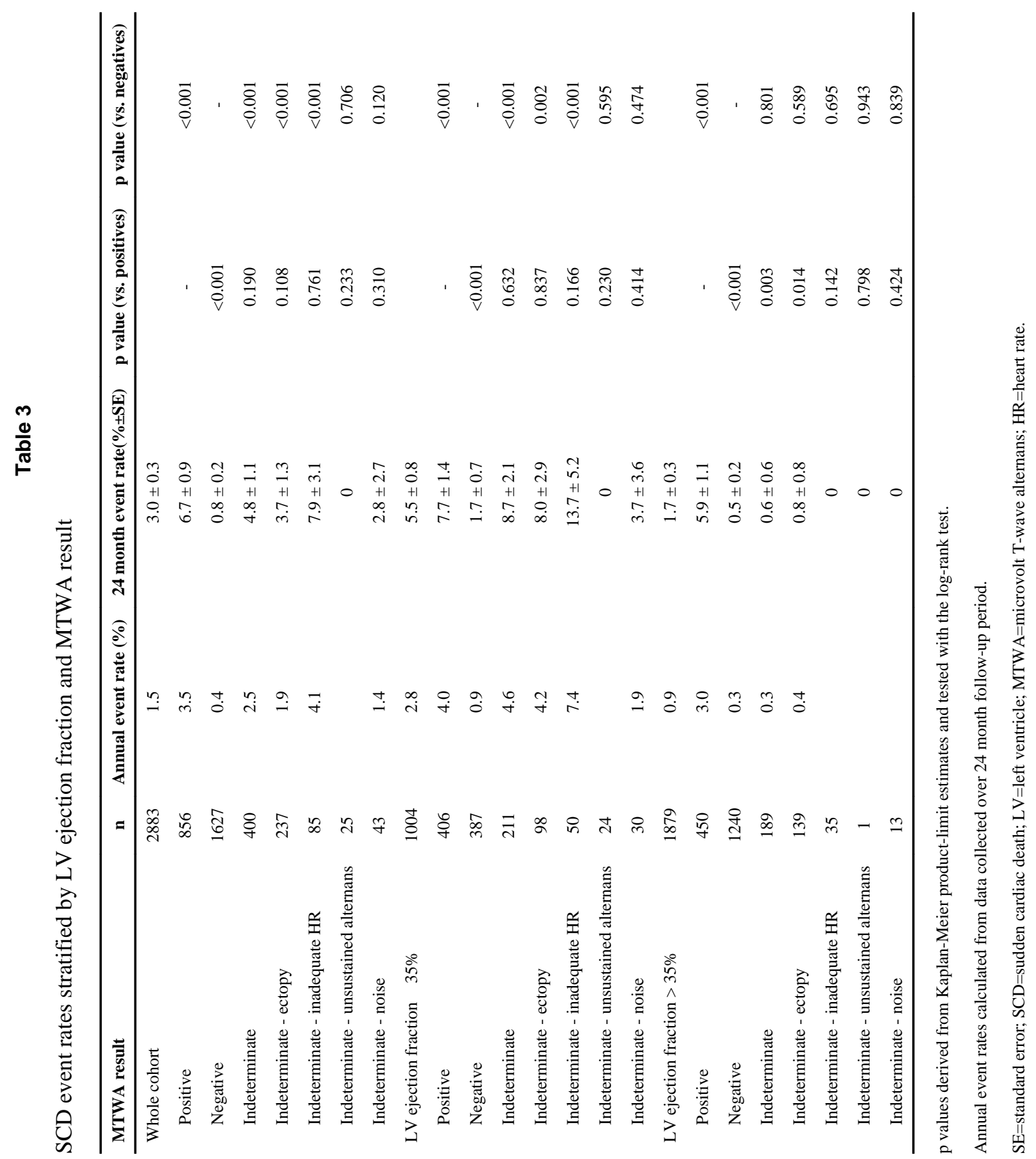

Heart Rhythm. Author manuscript; available in PMC 2013 August 01. 
